\title{
In vitro Study of the Antagonistic Effect of Low-dose Liquiritigenin on Gemcitabine-induced Capillary Leak Syndrome in Pancreatic Adenocarcinoma via Inhibiting ROS- Mediated Signalling Pathways
}

\author{
Wei Wu, Qing Xia, Rui-Jie Luo, Zi-Qi Lin, Ping Xue*
}

\begin{abstract}
Background: To investigate in-vitro antagonistic effect of low-dose liquiritigenin on gemcitabine-induced capillary leak syndrome (CLS) in pancreatic adenocarcinoma via inhibiting reactive oxygen species (ROS)mediated signalling pathways. Materials and Methods: Human pancreatic adenocarcinoma Panc-1 cells and human umbilical vein endothelial cells (HUVECs) were pre-treated using low-dose liquiritigenin for $24 \mathrm{~h}$, then added into gemcitabine and incubated for $48 \mathrm{~h}$. Cell viability, apoptosis rate and ROS levels of Panc-1 cells and HUVECs were respectively detected through methylthiazolyldiphenyl-tetrazoliumbromide (MTT) and flow cytometry. For HUVECs, transendothelial electrical resistance (TEER) and transcellular and paracellular leak were measured using transwell assays, then poly (ADP-ribose) polymerase 1 (PARP-1) and metal matrix proteinase-9 (MMP9) activity were assayed via kits, mRNA expressions of p53 and Rac-1 were determined through quantitative polymerase chain reaction (qPCR); The expressions of intercellular adhesion molecule 1 (ICAM-1), vascular cell adhesion molecule-1 (VCAM-1) and PARP-1 were measured via western blotting. Results: Low-dose liquiritigenin exerted no effect on gemcitabine-induced changes of cell viability, apoptosis rate and ROS levels in Panc-1 cells, but for HUVECs, liquiritigenin ( $3 \mu \mathrm{M})$ could remarkably elevate gemcitabineinduced decrease of cell viability, transepithelial electrical resistance (TEER), pro-MMP9 level and expression of ICAM-1 and VCAM-1 $(p<0.01)$. Meanwhile, it could also significantly decrease gemcitabine-induced increase of transcellular and paracellular leak, ROS level, PARP-1 activity, Act-MMP9 level, mRNA expressions of p53 and Rac-1, expression of PARP-1 and apoptosis rate $(p<0.01)$. Conclusions: Low-dose liquiritigenin exerts an antagonistic effect on gemcitabine-induced leak across HUVECs via inhibiting ROS-mediated signalling pathways, but without affecting gemcitabine-induced Panc-1 cell apoptosis. Therefore, low-dose liquiritigenin might be beneficial to prevent the occurrence of gemcitabine-induced CLS in pancreatic adenocarcinoma.
\end{abstract}

Keywords: liquiritigenin - gemcitabine - capillary leak syndrome - pancreatic adenocarcinoma - reactive oxygen

Asian Pac J Cancer Prev, 16 (10), 4369-4376

\section{Introduction}

Gemcitabine (Gemcitabine), a synthetic pyrimidine nucleoside, could block DNA synthesis and inhibit RNA synthesis to some extent. It is used as the standard chemotherapy for pancreatic adenocarcinoma in stage II, III and IV, although it's primarily palliative (Lau et al., 2012). As a severe result of the attack by gemcitabineinduced cytotoxicity for capillary endothelial cells (CECs), capillary leak syndrome (CLS) has been reported in several cases (De Pas et al., 2001; Pulkkanen et al., 2003; Biswas et al., 2004; Dahan et al., 2007). CLS, a serious disorder characterized by capillary hyperpermeability and albumin leak, is initiated by damaging CECs and their conjunctions that lead to dysfunction of capillary endothelial barrier (Stein et al., 2012). Clinically, administration of glucocorticoids and fluid resuscitation as well as discontinuation of gemcitabine can potentially reduce the mortality of CLS (Casadei et al., 2013). Nevertheless, Gemcitabine-induced cytotoxicity to CECs shows non-dose-dependence and individual difference. Progressive disease in some cases maintains a rapid development, which quickly results in ischemia-reperfusion injury and causes multiple organ failure (Stein et al., 2012), but at this time, the effect of all treatments on reversing progression is still limited. Hence, we proposed that prior-preventive measures should be implemented to prevent the occurrence of gemcitabineinduced CLS. Analysis on the action mechanism for gemcitabine indicated that two active metabolites of 
gemcitabine injured mitochondria to cause massive generation and transmembrane transport of ROS, and then that triggered ROS-mediated signalling pathways to induce the damage to cells in proliferative cycles (Chen et al., 2014). We inferred that gemcitabine-induced dysfunction of capillary endothelial barrier was primarily initiated by the downstream events in ROS-mediated signal transduction pathways. Accordingly, regulation or inhibition of ROS-mediated signalling pathways may potentially be the pivotal target and direction of the treatment to gemcitabine-induced CLS.

Liquiritigenin (LQ), a 4',7-dihydroxyflavanone generally existing in numerous plants, is mainly extracted from Glycyrrhiza uralensis Fisch. It is shown that LQ exerts the cytoprotective effects against ROS-induced cell injure (Choi et al., 2014). Phenolic hydroxyl with longer bond length in flavonoids molecules is more likely easy to trigger hydrogen abstraction reaction, and then the lost hydrogen atom is transferred and combined to ROS so as to terminate the ROS-mediated chain reaction (Singh et al., 2014). As shown in Figure 1 from PubChem, it is displayed in LQ of 2D structure and 3D conformer that phenolic hydroxyl of bond length at $\mathrm{C} 7$ is slightly longer than at $\mathrm{C} 4$, which may play a major role in the scavenging of ROS. The results from previous studies indicated that low dose of LQ could scavenge intracellular ROS and also down-regulate ROS-mediated plasminogen activator inhibitor 1 (PAI-1) expression to protect against ROSmediated human umbilical vein endothelial cells (HUVEs) injury (Xie et al., 2012). However, it is still unclear in some fields regarding whether low dose of LQ can antagonize gemcitabine-induced and ROS-mediated CLS as well as its intracellular mechanism.

Permeability of microvascular endothelium to fluids and proteins is regulated via the transcellular and paracellular leak across microvascular endothelium (Armstrong et al., 2012). In the state of oxidative stress, ROS-mediated cascade activation reaction cleavage $92 \mathrm{kDa}$ pro matrix metalloproteinase-9 (pro-MMP9, positions: 60-707) at the Arg106-Phe107 peptide bonds to produce the active 82-kDa MMP9 (Act-MMP9, positions: 60-707) that degrades the extracellular matrix (ECM), including intercellular adhesion molecule-1 (ICAM-1) and vascular cell adhesion molecule-1 (VCAM-1) to induce paracellular leak (Vacas et al., 2013). Simultaneously, a dramatic increase of intracellular ROS leads to $\mathrm{Ca}^{2+}$ influx into cells to activate the expression and transcription of ras-related $\mathrm{C} 3$ botulinum toxin substrate 1 (Rac-1) that increases transcellular leak (Axelsson et al., 2013). Finally, the poly (ADP-ribose) polymerase 1 (PARP-1) and tumor protein p53 (p53) are activated via ROS-

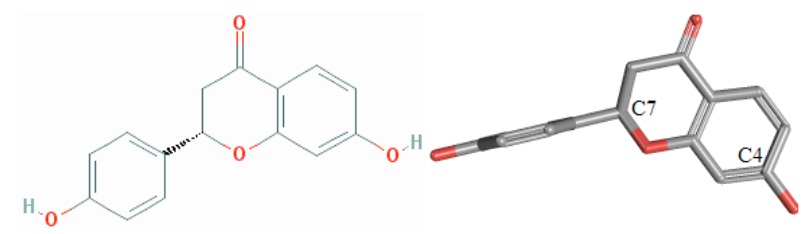

(A)

(B)

Figure 1. Phenolic Hydroxyl of Bond Length at C7 Slightly Longer than at C4. (A) 2D structure of LQ from PubChem; (B) 3D conformer of LQ from PubChem mediated signalling pathways to induce the apoptosis, further leading to increase of capillary leak (Montero et al., 2013; Wu et al., 2013). In this study, the antagonistic effect of low-dose LQ on gemcitabine-induced leak across HUVECs was researched via regulation or inhibition of ROS-mediated signal pathway, and the potential mechanism was also investigated.

\section{Materials and Methods}

\section{Sample source}

HUVECs and Panc-1 cells were obtained from Molecular Biology Lab of Sichuan University (Chengdu, China). Panc-1 cells were cultured in DMEM (Mediatech, VA, USA) with $10 \%$ fetal bovine serum (FBS), $100 \mathrm{U} /$ $\mathrm{mL}$ penicillin and $100 \mathrm{mg} / \mathrm{mL}$ streptomycin (SigmaAldrich, MO, USA); HUVECs were cultured in DMEM medium with $10 \%$ FBS. All cells were incubated at $37^{\circ} \mathrm{C}$ in humidified air with $5 \% \mathrm{CO}_{2}$.

\section{Methods}

MTT (methylthiazolyldiphenyl-tetrazoliumbromide) assay: Briefly, Panc- 1 cells and HUVECs were respectively seeded at a density of $2 \times 10^{5} / \mathrm{mL}$ cells in 96 -well plates (Chemicell GmbH, Berlin, Germany) and incubated for $24 \mathrm{~h}$. Then, both PANC-1 cells and HUVECs were respectively divided into the following groups according to our research targets: (1) blank-control group (blankCTRL) treated without gemcitabine (Eli Lilly, IN, USA) or LQ (Ark Pharm, Inc., IL, U.S.A); (2) negative-control group (negative-CTRL) treated with $5 \mu \mathrm{M}$ gemcitabine; (3) LQ-treated group 1 (LQ-1) treated with $5 \mu \mathrm{M}$ gemcitabine+0.03 $\mu \mathrm{M}$ LQ; (4) LQ-treated group 2 (LQ2) treated with $5 \mu \mathrm{M}$ gemcitabine+0.3 $\mu \mathrm{M}$ LQ; (5) LQtreated group 3 (LQ-3) treated with $5 \mu \mathrm{M}$ gemcitabine+3 $\mu \mathrm{M}$ LQ. Cells in each group occupied quintuple wells. The cells in blank control group were incubated in DMEM medium with $10 \%$ FBS for $48 \mathrm{~h}$, but without treatment with gemcitabine or LQ. The cells in negative control group were treated with $5 \mu \mathrm{M}$ gemcitabine and incubated for $48 \mathrm{~h}$. The cells in LQ-treated groups were separately pre-treated with different concentrations of LQ $(0.03,0.3$ and $3 \mu \mathrm{M})$ and incubated for $24 \mathrm{~h}$, and then which were added to $5 \mu \mathrm{M}$ of gemcitabine and co-incubated for $48 \mathrm{~h}$. After 48-h incubation, $20 \mu \mathrm{L}$ MTT (Sigma-Aldrich, MO, USA) $(5 \mathrm{mg} / \mathrm{mL})$ was added to each well and incubated for $4 \mathrm{~h}$. The absorbance value of each well was measured using Synergy Neo Multi-Mode Reader (Biotek, VT, USA) at a wavelength of $570 \mathrm{~nm}$. The cell viability rate was calculated according to the previous studies (YongHua et al., 2013).

Apoptosis assay: According to the result of MMT assay, $3 \mu \mathrm{M}$ of LQ exhibited a significantly protective effect against gemcitabine-induced injure for HUVECs, therefore, in our research, $3 \mu \mathrm{M}$ was designed as the effective concentration of LQ for treating with Panc-1 cells and HUVECs. Briefly, both Panc- 1 cells and HUVECs were separately divided into three groups: (1) blankcontrol group (blank-CTRL) treated without gemcitabine or LQ; (2) negative-control group (negative-CTRL) treated with $5 \mu \mathrm{M}$ gemcitabine; (3) LQ-treated group 3 
(LQ-3) treated with $5 \mu \mathrm{M}$ gemcitabine+3 $\mu \mathrm{M}$ LQ. Cells in each group occupied quintuple wells. The Panc- 1 cells and HUVECs of these groups were treated as described in MTT assay. All cells were collected and washed twice with PBS. And then cell apoptosis was analyzed by annexin V-FITC/PI apoptosis detection kit (ABCAM, Cambridge, UK) following the manufacturer's instructions, and was analyzed using flow cytometry system (Beckman Coulter, CA, USA). Each experiment was performed in triplicate.

Transwell assays: HUVECs were seeded on 24 mm transwell ${ }^{\circledR}$ with $0.4 \mu \mathrm{m}$ pore polyester membrane insert, sterile (Corning, MA, USA) and grown until confluence, and 3 days later, transepithelial electrical resistance (TEER) was stabilized at the values $>20$ $\Omega \times \mathrm{cm}^{2}$. Briefly, the HUVECs were grouped and treated as described in apoptosis assay. HUVECs' monolayer TEER were measured by Millicell ${ }^{\circledR}$ electrical resistance apparatus (Millipore, FL, USA) according to the manufacturer's instructions, and TEER values were calculated in accordance with the formula as described previously (Srinivasan et al., 2015), but the change of TEER apparatus could lead to the obvious differences of TEER values in some experiments. To further measure transcytosis, $0.4 \mathrm{mg} / \mathrm{mL}$ of fluorescein isothiocyanate (FITC)-conjugated albumin (Arista Biologicals, PA, USA) was added to endothelial monolayers for $10 \mathrm{~min}$ at $4^{\circ} \mathrm{C}$. The monolayers were washed by cold media twice to remove unbound albumin, and then incubated at $37^{\circ} \mathrm{C}$ for $2 \mathrm{~h}$. The aliquots of lower and upper chambers were measured in a Spectramax Gemcitabineini EM (SigmaAldrich, MO, USA). To further measure paracellular permeability, FITC-dextran $(50 \mathrm{~g} / \mathrm{mL})$ was added to the upper chambers of the transwell and co-incubated with cell monolayer at $37^{\circ} \mathrm{C}$ for $60 \mathrm{~min}$, then the samples were extracted from upper and lower chambers for fluorescence determination at an excitation wavelength of $485 \mathrm{~nm}$ and an emission wavelength of $535 \mathrm{~nm}$. And then, the permeability to dextran or albumin was calculated as described previously (Armstrong et al., 2012).

Cytoplasmic ROS detection: Panc-1 cells and HUVECs were grouped and treated as described in apoptosis assay. Then the cells were washed with PBS and incubated with 5 $\mu \mathrm{m}$ CellROX ${ }^{\circledR}$ Deep Red reagent (Life Technologies, NY, USA) for $30 \mathrm{~min}$ at $37^{\circ} \mathrm{C}$. Cytoplasmic ROS levels were measured by flow cytometry system (Beckman Coulter) according to the manufacturer's specification, whose value was shown as the mean fluorescence intensity value.

PARP-1 activity assay: HUVECs were grouped and treated as described in apoptosis assay, and gently washed twice with $200 \mu \mathrm{L}$ ice-cold Dulbecco's phosphate buffered saline (D-PBS). $100 \mu \mathrm{L}$ of cell lysis buffer was added to each well and incubated on ice for $30 \mathrm{~min}$ following a tapping at 5-minute intervals. Then, the lysate of each sample was extracted to a pre-cooling centrifuge tube and centrifuged for 10 seconds. The protein of each sample was quantified through BCA protein quantification kit (Abcam, FL, UK). After that, cell lysate samples were standardized at $20 \mathrm{ng}$ of protein. PARP-1 activity was measured by PARP-1 activity assay kit (GENMED, Shanghai, China) according to the manufacturer's instructions. A standard curve was made via a calibration standard. $20 \mathrm{ng}$ of protein was used to represent the total PARP-1 activity.

MMP9 activity assay: HUVECs were grouped and treated as described in apoptosis assay. MMP9 activity was measured with Gelatin-Zymography Kit (COSMO BIO CO., Tokyo, Japan) according to the manufacturer's instructions. The cultured supernatants of each group was collected and electrophoresed in gelatin at $15 \mathrm{~mA}$ for 90 min. $40 \mathrm{~mL}$ of SimplyBlue Safestain (Life Technologies, NY, USA) was added after renaturing the gel for each sample and incubated for $1 \mathrm{~h}$ at room temperature with gentle agitation. After staining, the gel of each group was scanned with a resolution of $300 \mathrm{dpi}$. The densitometry of each sample was analyzed by ImageJ software.

Quantitative real-time PCR (qPCR): HUVECs were grouped and treated as described in apoptosis assay. Total RNA was isolated from HUVECs $\left(1.0 \times 10^{6}\right.$ cells $)$ using RNeasy Plus Mini Kit (Qiagen, HRB, Germany). $5 \mu \mathrm{L}$ of total RNA was reverse-transcribed to cDNA using qScript SuperScript ${ }^{\circledR}$ VILO cDNA Synthesis Kit and Master Mix (Life Technologies, NY, USA). Real-time RT-PCR was implemented by SYBR ${ }^{\circledR}$ Green master mixes (Life Technologies, NY, USA). The real-time primers were designed using Primer Express ${ }^{\circledR}$ Software v3.0.1 License (Life Technologies, NY, USA). Primer sequences were as follows: p53 forward (5'-AGGCCTAACTCAAGGAT-3') and reverse (5'-CCCTTTTTGGACTTCAGGTG-3'); Rac-1 forward (5'-ATAGGCCCAGATTCACTGGTT-3') and reverse (5'-GAGACGGAGCTGTTGGTAAAA-3'); GAPDH forward (5'-CACTGCCACTCAGAAGACT-3') and reverse (5'- ACATTGGGGGTAGGAACAC-3'). Real-time PCR reactions were performed using SsoFast Eva Green Supermix (BioRad, CA, USA). PCR programme was designed as follows: $95^{\circ} \mathrm{C}$ for $60 \mathrm{~s}$, totally 40 cycles $\left(95^{\circ} \mathrm{C}\right.$ for $15 \mathrm{~s}, 60^{\circ} \mathrm{C}$ for $\left.15 \mathrm{~s}\right), 72^{\circ} \mathrm{C}$ for $45 \mathrm{~s}$ and $80^{\circ} \mathrm{C}$ for $5 \mathrm{~s}$ and the outcome was analyzed by Synergy Neo Multi-Mode Reader (Biotek).

Western blot: HUVECs were grouped and treated as described in apoptosis assay. Western blot was performed as described previously (Taylor et al., 2014). In brief, the cell lysates of each sample were acquired via lysis buffer, $20 \mathrm{ug}$ of total protein was separated by $10 \%$ acrylamide SDS-PAGE and transferred onto Immobilon ${ }^{\circledR}$ PVDF membranes (Sigma-Aldrich, MO, USA). Then the membranes were blocked, and incubated with specific primary antibodies in $4^{\circ} \mathrm{C}$ at overnight (1: 1000 of dilution) followed by incubation with a secondary antibody. Membranes-bind antibodies were tested by Amersham ${ }^{\mathrm{TM}}$ ECL $^{\mathrm{TM}}$ Western Blotting Reagent Pack (Sigma-Aldrich, MO, USA) and the outcome was analyzed by ImageJ software.

\section{Statistical data analysis}

SPSS 19.0 statistical software was used for data analysis. Statistical evaluations were performed by independent-samples $t$ test, expressed by the mean \pm standard deviation $(\mathrm{x} \pm \mathrm{s}) . p<0.05$ was considered statistically significant.

\section{Results}

Effect of low-dose LQ on the gemcitabine-induced 
decrease in cell viability of Panc- 1 cells and HUVECs

For Panc-1 cells, gemcitabine significantly decreased cell viability in negative-control group $(40.33 \% \pm 1.52 \%)$ compared with blank-control group $(98.76 \% \pm 1.30 \%$, $\left.{ }^{\# \#} p<0.01\right)$, but there was no statistical significance between low-dose LQ-treated groups and negative-control group regarding the cell viability $\left({ }^{\triangle} p>0.05\right)$ (Figure $\left.2 \mathrm{~A}\right)$. For HUVECs, gemcitabine evidently reduced the cell viability in negative-control group $(66.08 \% \pm 3.26 \%)$ compared with blank-control group $\left(99.12 \% \pm 1.26 \%,{ }^{\# \#} p<0.01\right)$. However, compared with negative-control group, LQ of 0.3 and 3 $\mu \mathrm{M}$ obviously elevated the cell viability respectively in LQ-2 group $(76.33 \% \pm 4.49 \%, * p<0.05)$ and LQ-3 group $(85.33 \% \pm 1.69 \%, * * p<0.01)$. There was no statistical significance between negative-control group and LQ-1 group with regard to the cell viability $\left({ }^{\triangle} p>0.05\right)$ (Figure 2B).

Effect of low-dose LQ on the gemcitabine-induced increase in the apoptosis rate of Panc-1 cells and HUVECs

For Panc-1 cells, gemcitabine induced the increase of cell apoptosis rate in negative-control group $(48.3 \% \pm 2.6 \%)$ compared with blank-control group $(1.6 \% \pm 0.8 \%$, $\left.{ }^{\# \#} p<0.01\right)$, whereas the apoptosis rate in LQ-3 group $(45.8 \% \pm 3.1 \%)$ was shown no statistical significance
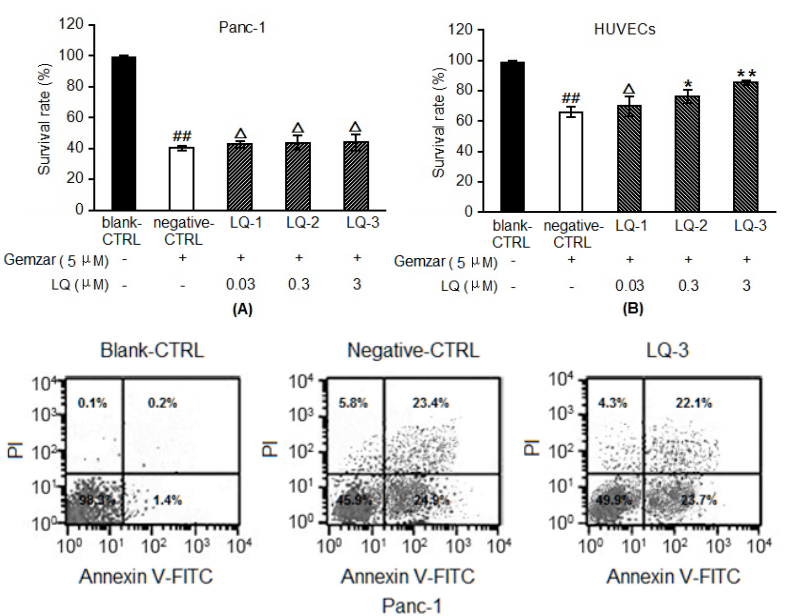

(C)
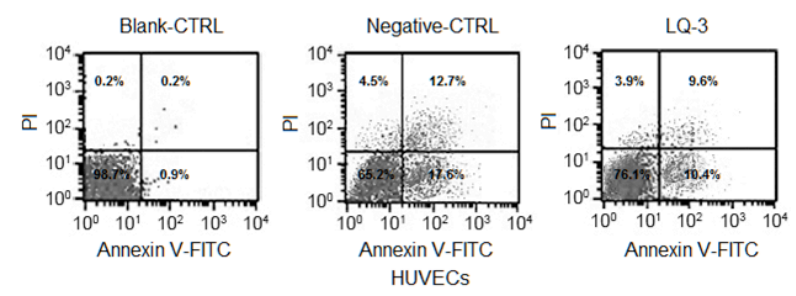

(D)

Figure 2. Comparison between Panc-1 Cells and HUVECs (Low-dose LQ was Shown different Effects on Gemcitabine-Induced Changes of Cell Viability and Apoptosis Rate).(A) Low-dose LQ didn't affect gemcitabineinduced decrease in the cell viability of Panc-1 cells; (B) 0.3 and $3 \mu \mathrm{M}$ of LQ significantly ameliorated gemcitabine-induced decrease in the cell viability of HUVECs; (C) $3 \mu \mathrm{M}$ LQ didn't affect gemcitabine-induce increase in the apoptosis rate of Panc1 cells; (D) $3 \mu \mathrm{M}$ LQ remarkably reduced gemcitabine-induce increase in the apoptosis rate of HUVECs. ${ }^{\# \#} p<0.01 \mathrm{vs}$. blankCTRL, ${ }^{\triangle} p>0.05 v s$. negative-CTRL, ${ }^{*} p<0.05$ and ${ }^{*} p p<0.01$ vs. negative-CTRL compared with negative-control group $\left({ }^{\triangle} \mathrm{p}>0.05\right)$ (Figure 2C). For HUVECs, gemcitabine remarkably increased the cell apoptosis rate in negative-control group $(30.3 \% \pm 1.6 \%)$ compared with blank-control group $\left(1.1 \% \pm 0.5 \%,{ }^{\# \#} p<0.01\right)$, but LQ of $3 \mu \mathrm{M}$ significantly decreased the apoptosis rate in LQ-3 group $(20.0 \% \pm 2.2 \%)$ compared to with negative-control group ( $\left.{ }^{\#} p<0.01\right)$ (Figure 2D).

Effect of low-dose LQ on the gemcitabine-induced changes of transcellular and paracellular leak as well as TEER in HUVECs

Gemcitabine-induced the distinct decrease in the TEER $\left(\Omega \times \mathrm{cm}^{2}\right)$ in HUVECs at $6,12,24$ and $48 \mathrm{~h}$ in negative-control group compared with blank-control group $\left({ }^{\# \#} p<0.01\right)$, but compared with negative-control

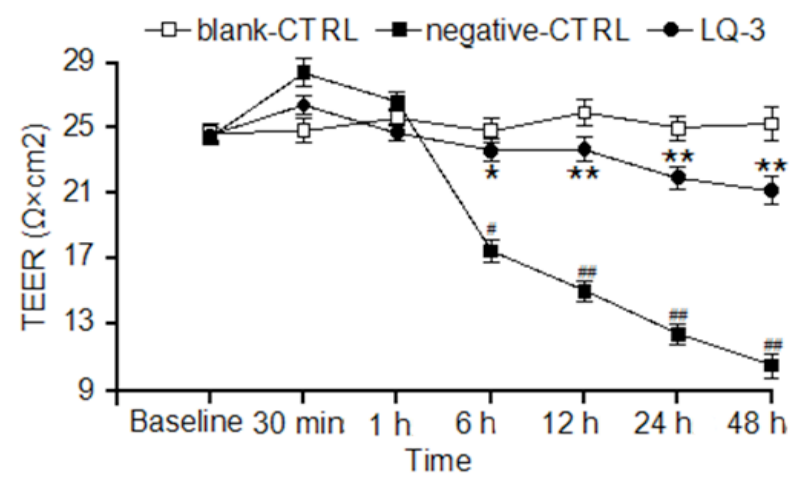

(A)

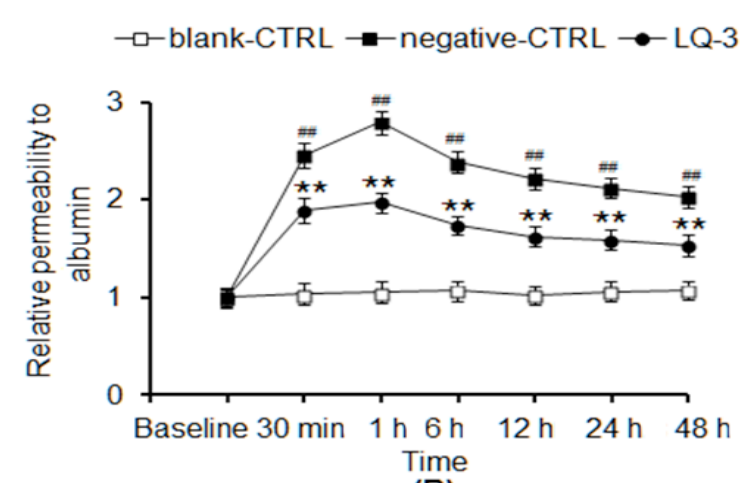

(B)

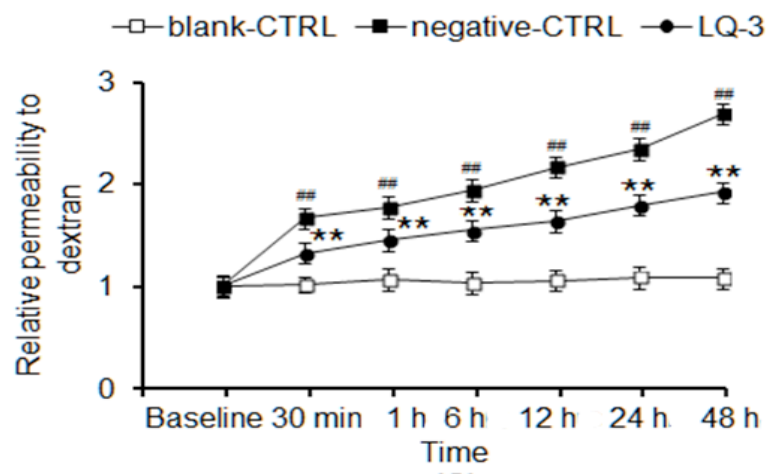

(C)

Figure 3. Low-dose LQ Reversed Gemcitabine-Induced Hyperpermeability in HUVECs. (A) Low-dose LQ was shown protective activity against gemcitabine-induced decrease in TEER; (B) $3 \mu \mathrm{M}$ LQ significantly ameliorated gemcitabineinduced increase of the transcellular leak; (C) $3 \mu \mathrm{M} \mathrm{LQ}$ visibly reduced gemcitabine-induced increase of the paracellular leak. ${ }^{\# \# p} p<0.01 v s$. blank-CTRL, ${ }^{* *} p<0.01 v s$. negative-CTRL 
group, $3 \mu \mathrm{M} L Q$ remarkably increased the TEER in LQ-3 group $(* * p<0.01)$ (Figure $3 \mathrm{~A})$. Gemcitabine remarkably induced the increase in the relative permeability to albumin at $30 \mathrm{~min}(2.45 \pm 0.12$ vs. $1.03 \pm 0.11), 1 \mathrm{~h}(2.79 \pm 0.12 \mathrm{vs}$. $1.05 \pm 0.10), 6 \mathrm{~h}(2.38 \pm 0.11$ vs. $1.07 \pm 0.10), 12 \mathrm{~h}(2.21 \pm 0.12$ vs. $1.02 \pm 0.09), 24 \mathrm{~h}(2.12 \pm 0.10$ vs. $1.06 \pm 0.09), 48 \mathrm{~h}$ $(2.03 \pm 0.11$ vs. $1.07 \pm 0.09)$ in negative-control group compared with blank-control group $\left.{ }^{\# \#} p<0.01\right)$. However, compared with negative-control group, $3 \mu \mathrm{MLQ}$ evidently decreased the relevant permeability to albumin in LQ-3 group at $30 \mathrm{~min}(1.89 \pm 0.12, * * p<0.01), 1 \mathrm{~h}(1.97 \pm 0.10$, $* * p<0.01)$, 6h $(1.74 \pm 0.09, * * p<0.01), 12 \mathrm{~h}(1.62 \pm 0.10$, $* * p<0.01), 24 \mathrm{~h}(1.59 \pm 0.10, * * p<0.01), 48 \mathrm{~h}(1.53 \pm 0.11$, $* * p<0.01$ ) (Figure 3B). Gemcitabine obviously increased the relevant permeability to dextran at $30 \mathrm{~min}(1.67 \pm 0.09$ vs. $1.02 \pm 0.07), 1(1.78 \pm 0.11$ vs. $1.07 \pm 0.10), 6 \mathrm{~h}(1.94 \pm 0.11$ vs. $1.06 \pm 0.10), 12 \mathrm{~h}(2.17 \pm 0.11$ vs. $1.06 \pm 0.10), 24 \mathrm{~h}$ $(2.34 \pm 0.11$ vs. $1.09 \pm 0.11), 48 \mathrm{~h}(2.69 \pm 0.11$ vs. $1.08 \pm 0.10)$ in negative-control group compared with blank-control group ( ${ }^{\# \#} p<0.01$ ). However, compared with negativecontrol group, $3 \mu \mathrm{M}$ LQ significantly induced the decrease in the relevant permeability to dextran in LQ-3 group at $30 \min (1.33 \pm 0.11, * * p<0.01), 1 \mathrm{~h}\left(1.46 \pm 0.10,{ }^{*} * p<0.01\right)$, 6h $(1.55 \pm 0.10, * * p<0.01), 12 \mathrm{~h}(1.64 \pm 0.11, * * p<0.01)$, $24 \mathrm{~h}(1.80 \pm 0.11, * * p<0.01), 48 \mathrm{~h}(1.92 \pm 0.11, * * p<0.01)$ (Figure 3C).

Effect of low-dose LQ on the gemcitabine-induced increase in ROS levels of Panc-1 cells and HUVECs

For Panc-1 cells, gemcitabine significantly increased intracellular ROS levels at $30 \mathrm{~min}(7.06 \pm 0.32 \mathrm{vs}$. $1.61 \pm 0.31), 1 \mathrm{~h}(8.68 \pm 0.35$ vs. $1.69 \pm 0.29), 6 \mathrm{~h}(9.61 \pm 0.29$ vs. $1.89 \pm 0.30), 12 \mathrm{~h}(8.72 \pm 0.33$ vs. $1.59 \pm 0.29), 24 \mathrm{~h}$ $(8.56 \pm 0.37$ vs. $1.75 \pm 0.36), 48 \mathrm{~h}(7.84 \pm 0.34$ vs. $1.88 \pm 0.35)$ in negative-control group compared with blank-control group $\left({ }^{\# \#} p<0.01\right)$, but ROS levels in LQ-treated groups was shown no statistical significance compared with negativecontrol group $\left({ }^{\triangle} \mathrm{p}>0.05\right)$ (Figure 4A). For HUVECs, gemcitabine distinctly elevated intracellular ROS levels at $30 \mathrm{~min}(3.63 \pm 0.19$ vs. $1.23 \pm 0.17), 1 \mathrm{~h}(4.39 \pm 0.16 \mathrm{vs}$. $1.22 \pm 0.15), 6 \mathrm{~h}(4.76 \pm 0.18$ vs. $1.18 \pm 0.16), 12 \mathrm{~h}(4.47 \pm 0.18$ vs. $1.29 \pm 0.16), 24 \mathrm{~h}(4.42 \pm 0.19$ vs. $1.14 \pm 0.17), 48 \mathrm{~h}$ $(4.29 \pm 0.19$ vs. $1.29 \pm 0.17)$ in negative-control group compared with blank-control group $\left.{ }^{\# \#} p<0.01\right)$. However, compared with negative-control group, $3 \mu \mathrm{M}$ LQ remarkably decreased ROS levels in LQ-3 group at 30 $\min (2.26 \pm 0.15, * * p<0.01)$, 1h $(2.65 \pm 0.14, * * p<0.01)$, $6 \mathrm{~h}(2.78 \pm 0.15, * * p<0.01), 12 \mathrm{~h}(2.33 \pm 0.17, * * p<0.01)$, 24h $(2.27 \pm 0.16, * * p<0.01), 48 \mathrm{~h}(2.09 \pm 0.15, * * p<0.01)$ (Figure 4B).

Antagonizing gemcitabine-induced apoptosis in HUVECs via repression of low-dose $L Q$ to PARP-1 and p53

In negative-control group, gemcitabine evidently induced the increase of PARP-1 activity and expression as well as the upregulation of p53 mRNA expression by comparison to blank-control group $\left.{ }^{\# \#} p<0.01\right)$. However, in LQ-3 group, $3 \mu \mathrm{M}$ LQ obviously decreased the activity and expression of PARP-1 and the expression of p53 mRNA compared with negative-control group $(* * p<0.01)$ (Figure 5A, 5B and 5D).
Alleviating gemcitabine-induced transcellular leak across HUVECs via inhibition of low-dose $L Q$ to Rac-1 mRNA expression

Gemcitabine dramatically increased Rac-1 mRNA expression in negative-control group by comparison to blank-control group $\left({ }^{\# \#} p<0.01\right)$. However, compared with negative-control group, $3 \mu \mathrm{M}$ LQ distinctly decreased Rac-1 mRNA expression in LQ-3 group $(* * p<0.01)$ (Figure 5D).

Alleviating gemcitabine-induced paracellular leak across HUVECs via repression of low-dose LQ to MMP9 activation for upregulating the expression of ICAM-1 and VCAM-1

Gemcitabine evidently increased Act-MMP9 level in negative-control group compared with blank-control group ${ }^{\# \#} p<0.01$ ), whereas compared with negative-control group, $3 \mu \mathrm{M}$ LQ obviously decreased Act-MMP9 level in LQ-3 group $(* * p<0.01)$. Meanwhile, the Pro-MMP9 level in negative-control group was dramatically lower than in blank-control group ("\# $p<0.01$ ), but that in LQ-3 group was distinctly higher than in negative-control group $(* * p<0.01)$ (Figure 5B). Gemcitabine evidently reduced the expression of ICAM-1 and VCAM-1 in negativecontrol group compared with blank-control group $\left.{ }^{\# \#} p<0.01\right)$. Nevertheless, compared with negative-control group, $3 \mu \mathrm{M}$ LQ elevated the expression of ICAM-1 and VCAM-1 in LQ-3 group significantly ( $* * p<0.01)$.

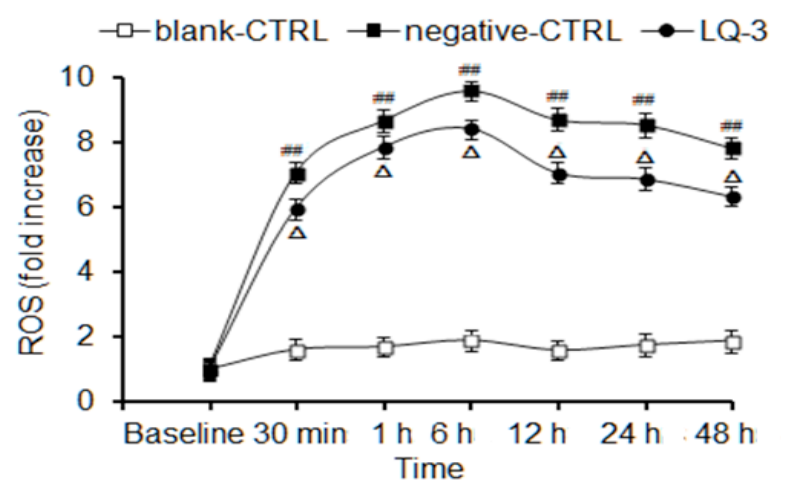

(A)

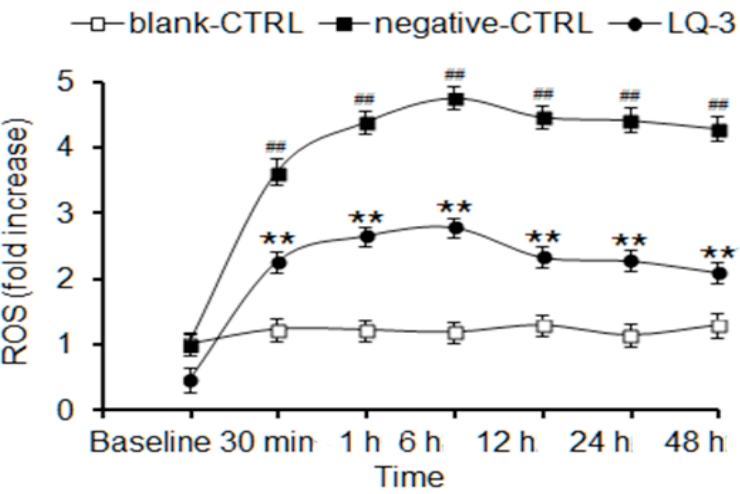

(B)

Figure 4. Effect of Low-dose LQ on the Gemcitabineinduced Increase in ROS Levels of Panc-1 Cells and HUVECs. (A) Low-dose LQ didn't affect gemcitabineinduced increase in ROS levels of Panc-1 cells; (B) $3 \mu \mathrm{M}$ LQ remarkably reduced the gemcitabine-induced increase in ROS levels of HUVECs. \#\#p<0.01 vs. blank-CTRL, ${ }^{* *} p<0.01 v s$. negative-CTRL, ${ }^{\triangleright} p>0.05 v s$. negative-CTRL 


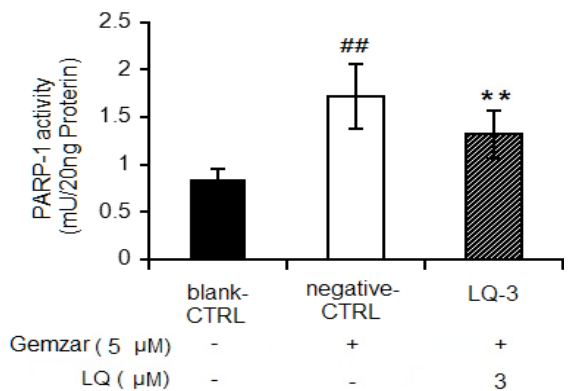

(A)

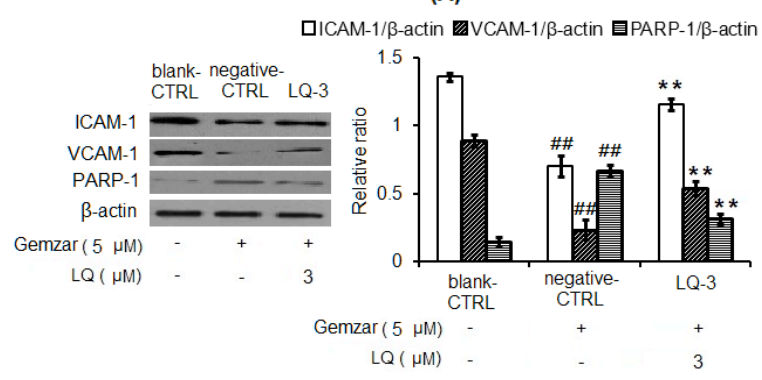

(B)

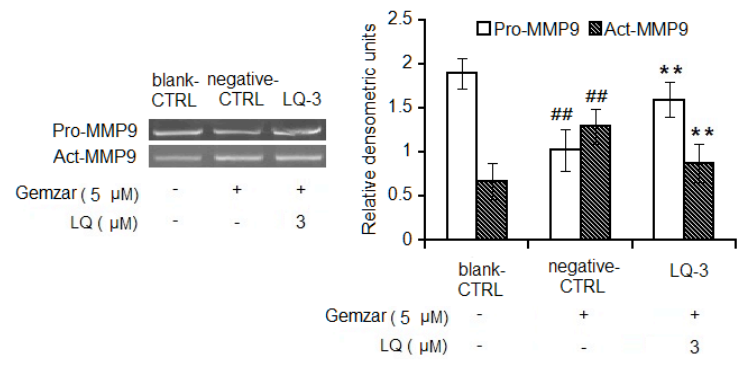

(C)

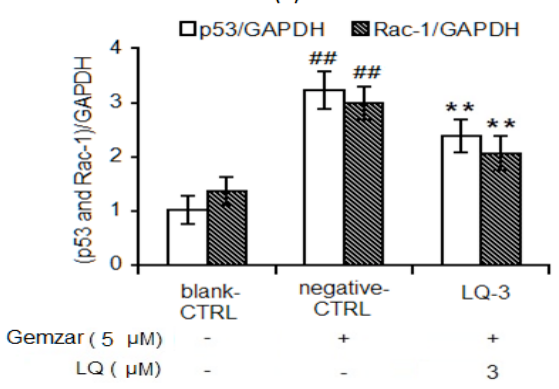

(D)

Figure 5. Potential Antagonistic Mechanisms of Lowdose LQ on Gemcitabine-induced Transcellular and Paracellular Leak and Apoptosis via Inhibition to ROS-mediated Signal Pathway in HUVECs. (A) Lowdose LQ repressed PARP-1 activity to antagonize gemcitabineinduced apoptosis; (B) Low-dose LQ was shown the inhibitory effects on gemcitabine-induced apoptosis and transcytosis by suppressing mRNA expression of p53 and Rac-1; (C) Low-dose LQ increased the expression of ICAM-1 and VCAM-1 to reduce gemcitabine-induced paracellular leak and decreased expression of PARP-1 to repress gemcitabine-induced apoptosis; (D) Lowdose LQ repressed MMP9 activity to attenuate gemcitabineinduced paracellular leak. ${ }^{\# \#} p<0.01$ vs. blank-CTRL, ${ }^{* *} p<0.01$ vs. negative-CTRL

\section{Discussion}

After being transported into cells, gemcitabine is conversed to two active metabolites including gemcitabine diphosphate (dFdCDP) and gemcitabine triphosphate (dFdCTP) by deoxycitidine kinase. dFdCDP inhibits ribonucleotide reductase and $\mathrm{dFdCTP}$ to compete with the deoxycytidine triphosphate and incorporate into DNA, which synergistically inhibit endonuclear and mitochondria DNA synthesis and RNA synthesis to some extent as well as induce DNA mutation and fragmentation, and then injure the mitochondrial respiratory chain to initiate massive production of ROS, impair the mitochondrial structural integrity and increase mitochondrial membrane permeability. After that, it can elevate mitochondrial proton-motive force $(\Delta \mathrm{P})$ to form electronic leak that triggers substantial ROS to transmembrane and is involved in ROS-mediated signalling pathways (Wong et al., 2013). Cell-cycle specificity of gemcitabine would induce cellular arrest at G1-S border, which means that the normal cells with proliferating cycle including CECs are likewise sensitive to gemcitabine, and also suffer the attack of gemcitabine-induced cytotoxicity (Matsuda, 1997; Dasanu, 2008). Consistent with our results, gemcitabine significantly increased the ROS level in Panc-1 cells and HUVECs, leading to the increased apoptosis rate and decreased cell viability. ROS-mediated cascade activation reaction might lead to dysfunction of capillary endothelial barrier, ultimately resulting in capillary hyperpermeability and albumin leak, which had been confirmed in our study according to the following results: Gemcitabine evidently decreased TEER of HUVECs monolayers and increased the transcellular and paracellular leak across HUVECs. For revealing the intracellular mechanism for ROS-induced capillary leak, our research indicated that gemcitabine obviously elevated MMP9 activity that decreased the expression of ICAM-1 and VCAM-1, which contributed for the increase of paracellular leak. Meanwhile, gemcitabine markedly increased the mRNA expression of Rac-1 to induce transcytosis, and the mRNA expression of p53 as well as activity and expression of PARP-1 to induce apoptosis.

There are growing evidences indicating that low-dose LQ could scavenge intracellular ROS and regulate or inhibit ROS-mediated signalling pathways to protect a series of cells including neurons, HUVECs, macrophages, hippocampal neurons, osteoblasts and hepatocytes (Pan et al., 2000; Xie et al., 2012; Yang et al., 2013; Suh et al., 2014; Park et al., 2015). However, most studies have indicated that reducing elevated ROS is resistant to gemcitabine for pancreatic adenocarcinoma cells (Ju et al., 2014; Suzuki et al., 2015; Wang et al., 2014; Cheng et al., 2014). Therefore, for pancreatic adenocarcinoma cells, application of preventive drugs, a preventive measure to prevent the occurrence of gemcitabine-induced CLS in chemotherapy for pancreatic adenocarcinoma, ought not to affect gemcitabine-induced increase in ROS as well as ROS-mediated downstream events. Our research results demonstrated that low-dose LQ met the requirements described above, as the following results: For Panc-1 cells, low-dose LQ was shown no effect on the gemcitabine-induced increase of ROS levels and apoptosis rate as well as decrease of cell viability. Due to more frequent replication of mitochondrial and endonuclear DNA in tumor cells of abnormal proliferation, gemcitabine with cell-cycle specificity is prone to damage more mitochondria (Liu et al., 2014). 
And relative-high ROS levels needed by tumor cells might damage the mitochondria and enhance the effect of gemcitabine-induced injury to mitochondria (Chen et al., 2014). Meanwhile, based on analysis of structureactivity relationship to flavonoid molecules, we found that the antioxidant activity of LQ was slightly lower than a few flavonoid molecules, such as isoliquiritigenin (Gaur et al., 2014; Peng et al., 2015). Accordingly, we inferred that low-dose LQ without affecting gemcitabine-induced increase of ROS in tumor cells was due to the following reasons: 1) Both of quantity in injured mitochondria and injured extent of mitochondria induced by gemcitabine in tumor cells are much high; 2) The scavenging ability of low-dose LQ on ROS is relatively low. On the other hand, the prior-preventive usage of low-dose LQ could effectively reduce gemcitabine-induced increase of ROS level in HUVECs. Compared with the tumor cells in abnormal proliferation cycle, the normal cells in normal proliferation cycle are relatively less sensitive to gemcitabine, probably leading to both of quantity in injured mitochondria and injured extent of mitochondria in normal cells induced by gemcitabine much lower than in tumor cells (Liu et al., 2013). Consequently, low-dose LQ only with the relatively lower ability to scavenge ROS could also effectively reduce the gemcitabine-induced increase of ROS in HUVECs. With the elimination of ROS in HUVECs, ROS-mediated changes in downstream events including activity of MMP9 and PARP-1, expression of ICAM-1, VCAM-1, Rac-1 and p53 had been effectively reversed. Accordingly, ROS-mediated decrease in TEER and cell viability was increased; ROS-mediated increase in transcellular and paracellular leak as well as apoptosis rate were reduced.

In conclusion, low-dose liquiritigenin exerts an antagonistic effect on gemcitabine-induced leak across HUVECs via inhibiting ROS-mediated signalling pathways, but without affecting gemcitabine-induced Panc-1 cell apoptosis. Therefore, low-dose liquiritigenin might be beneficial to prevent the occurrence of gemcitabine-induced CLS in pancreatic adenocarcinoma.

\section{Acknowledgements}

This study was supported by National Natural Science Foundation of China (No. 81072910) and Science \& Technology Support Program of Sichuan Province (N0. 2011SZ0291).

\section{References}

Axelsson J, Rippe A, Sverrisson K, et al (2013). Scavengers of reactive oxygen species, paracalcitol, RhoA, and Rac-1 inhibitors and tacrolimus inhibit angiotensin II-induced actions on glomerular permeability. Am J Physiol Renal Physiol, 5, 237-43.

Armstrong SM, Khajoee V, Wang C, et al (2012). Co-regulation of transcellular and paracellular leak across microvascular endothelium by dynamin and Rac. Am J Pathol, 180, 130823.

Biswas S, Nik S, Corrie PG (2004). Severe gemcitabine-induced capillary-leak syndrome mimicking cardiac failure in a patient with advanced pancreatic cancer and high-risk cardiovascular disease. Clin Oncol (R Coll Radiol), 16, 577-9.

Chen SH, Li DL, Yang F, et al (2014). Gemcitabine-induced pancreatic cancer cell death is associated with MST1/ cyclophilin D mitochondrial complexation. Biochimie, $103,71-9$.

Choi EM, Suh KS, Lee YS (2014). Liquiritigenin restores osteoblast damage through regulating oxidative stress and mitochondrial dysfunction. Phytother Res, 28, 880-6.

Cheng ZX, Wang DW, Liu T, et al (2014). Effects of the HIF-1 $\alpha$ and NF- $x \mathrm{~B}$ loop on epithelial-mesenchymal transition and chemoresistance induced by hypoxia in pancreatic cancer cells. Oncol Rep, 31, 1891-8.

Casadei Gardini A, Aquilina M, Oboldi D, et al (2013). Separate episodes of capillary leak syndrome and pulmonary hypertension after adjuvant gemcitabine and three years later after nab-paclitaxel for metastatic disease. $B M C$ Cancer, 13, 542 .

Dasanu CA (2008). Gemcitabine: vascular toxicity and prothrombotic potential. Expert Opin Drug Saf, 7, 703-16.

Dahan L, Ressiot E, Cournede A, et al (2007). Anasarca, a complication of chemotherapy with gemcitabine in two patients with pancreatic cancer. Gastroenterol Clin Biol, 31, 1143-5.

De Pas T, Curigliano G, Franceschelli L, et al (2001). Gemcitabine-induced systemic capillary leak syndrome. Ann Oncol, 12, 1651-2.

Gaur R, Yadav KS, Verma RK, et al (2014). In vivo anti-diabetic activity of derivatives of isoliquiritigenin and liquiritigenin. Phytomedicine, 21, 415-22.

Ju HQ, Gocho T, Aguilar M, et al (2014). Mechanisms of overcoming intrinsic resistance to gemcitabine in pancreatic ductal adenocarcinoma through the redox modulation. $\mathrm{Mol}$ Cancer Ther, [Epub ahead of print].

Liu DL, Bu HQ, Jin HM, et al (2014). Enhancement of the effects of gemcitabine against pancreatic cancer by oridonin via the mitochondrial caspase-dependent signaling pathway. $\mathrm{Mol}$ Med Rep, 10, 3027-34.

Liu Z, Li D, Zheng X, et al (2013). Selective induction of apoptosis: promising therapy in pancreatic cancer. $\mathrm{Curr}$ Pharm Des, 9, 2259-68.

Lau PC, Zheng SF, Ng WT, et al (2012). Inoperable pancreatic adenocarcinoma rendered complete remission by highintensity focused ultrasound concurrent with gemcitabinecapecitabine chemotherapy: case report and topic review. $J$ Dig Dis, 13, 60-4.

Montero J, Dutta C, van Bodegom D, et al (2013).p53 regulates a non-apoptotic death induced by ROS. Cell Death Differ, 20, 1465-74.

Matsuda A (1997). Nucleoside kinases and new types of antitumor nucleosides. Gan To Kagaku Ryoho, 24, 1594-9.

Peng F, Du Q, Peng C, et al (2015). A review: the pharmacology of isoliquiritigenin. Phytother Res, [Epub ahead of print].

Park SM, Ki SH, Han NR, et al (2015). Tacrine, an oral acetylcholinesterase inhibitor, induced hepatic oxidative damage, which was blocked by liquiritigenin through GSK3beta inhibition. Biol Pharm Bull, 38, 184-92.

Pulkkanen K, Kataja V, Johansson R (2003). Systemic capillary leak syndrome resulting from gemcitabine treatment in renal cell carcinoma: a case report. $J$ Chemother, 15, 287-9.

Pan X, Kong LD, Zhang Y, et al (2000). In-vitro inhibition of rat monoamine oxidase by liquiritigenin and isoliquiritigenin isolated from Sinofranchetia chinensis. Acta Pharmacol Sin, 21, 949-53.

Suzuki S, Okada M, Shibuya K, et al (2015). JNK suppression of chemotherapeutic agents-induced ROS confers 
Wei Wu et al

chemoresistance on pancreatic cancer stem cells. Oncotarget, 6, 458-70.

Srinivasan B, Kolli AR, Esch MB, et al (2015). TEER measurement techniques for in vitro barrier model systems. J Lab Autom, 20, 107-26.

Singh M, Kaur M, Silakari O (2014). Flavones: an important scaffold for medicinal chemistry. Eur J Med Chem, 84, 206-39.

Suh KS, Rhee SY, Kim YS, et al (2014). Protective effect of liquiritigenin against methylglyoxal cytotoxicity in osteoblastic MC3T3-E1 cells. Food Funct, 5, 1432-40.

Stein DM, Scalea TM, (2012). Capillary leak syndrome in trauma: what is it and what are the consequences? Adv Surg, 46, 237-53.

Taylor SC, Posch A (2014). The design of a quantitative western blot experiment. Biomed Res Int, 2014, 361590.

Vacas E, Bajo AM, Schally AV, et al (2013). Vasoactive intestinal peptide induces oxidative stress and suppresses metastatic potential in human clear cell renal cell carcinoma. Mol Cell Endocrinol, 365, 212-22.

Wang R, Cheng L, Xia J, et al (2014). Gemcitabine resistance is associated with epithelial-mesenchymal transition and induction of HIF-1 $\alpha$ in pancreatic cancer cells. Curr Cancer Drug Targets, 14, 407-17.

Wu X, Deng G, Li M, et al (2013). Wnt/ $\beta$-Catenin signaling reduces Bacillus Calmette-Guerin-induced macrophage necrosis through a ROS-mediated PARP/AIF-dependent pathway. BMC Immunol, 16, 16.

Wong BS, Yoong SL, Jagusiak A, et al (2013). Carbon nanotubes for delivery of small molecule drugs. Adv Drug Deliv Rev, 65, 1964-2015.

Xie X, Zhang HD, Chen YZ, et al (2012). Chemical constituents and activities of total flavonoids from Yushen Tang. Chinese Materia Medica, 37, 3585-90.

Yang EJ, Park GH, Song KS (2013). Neuroprotective effects of liquiritigenin isolated from licorice roots on glutamate-induced apoptosis in hippocampal neuronal cells. Neurotoxicology, 39, 114-23.

Zhan YH, Huang XF, Hu XB, et al (2013). Growth inhibition and apoptosis induction of human umbilical vein endothelial cells by apogossypolone. Asian Pac J Cancer Prev, 14, 1791-5. 